\title{
О ВЫБОРЕ КООРДИНАТНОЙ СИСТЕМЫ В МЕТОДЕ РИТЦА И В МЕТОДЕ ГАЛЕРКИНА ДЛЯ ЭВОЛЮЦИОННЫХ УРАВНЕНИЙ
}

\author{
(Представил А. Хумал)
}

Известно [ $\left.{ }^{1}\right]$, что при решении стационарного уравнения $A u=f$ методом Ритца, когда координатная система полна в области определения оператора $A$, невязка может и не сходиться при некоторой правой части $f$. В настоящей статье показано, что для любого неограниченного самосопряженного положительно определенного оператора существует координатная система такая, что невязка в методе Ритца сходится при любой правой части. Такие координатные системы позволяют доказать существование решения некоторых эволюционных уравнений и получить при их решении методом Галеркина двусторонние оценки сходимости.

1. Пусть $A$ - неограниченный самосопряженный положительно определенный оператор в сепарабельном гильбертовом пространстве $H$ с областью определения $D(A)$. Тогда уравнение

$$
A u=f
$$

при любом $f \in H$ имеет единственное решение $u=A^{-1} f \in D(A)$. Пусть задана координатная система $\left\{\varphi_{k}\right\}_{1}{ }^{\infty} \subset D(A)$. Обозначим через $H_{n}$ линейные оболочки элементов $\varphi_{1}, \ldots, \varphi_{n}$, а через $P_{n}-$ ортопроекторы в $H$, проектирующие на $H_{n}$.

Решение уравнения (1) методом Ритца $\left[{ }^{2}\right]$ (представляющим собой частный случай метода Галеркина) равносильно решению спроектированных уравнений

$$
P_{n} A u_{n}=P_{n} f, \quad u_{n} \in H_{n}, \quad n=1,2, \ldots .
$$

Решением спроектированного уравнения является $u_{n}=Q_{n} u$, где $Q_{n}$ - ортопроектор в энергетическом пространстве $H_{A}$, проектирующий на $H_{n}$. Значит, если координатная система полна в $H_{A}$, то $u_{n} \rightarrow u$ в $H_{A}$. Предположим, что координатная система полна в $D(A)$ с нормой $\|x\|_{D(A)}=\|A x\|, x \in D(A)$, и поставим вопрос о сходимости метода Ритца в $D(A)$, или, что равносильно, о сходимости невязки $A u_{n}-f \rightarrow 0$ в $H$. Отметим попутно, что существуют $f \in H$ и полная в $D(A)$ координатная система, при которых невязка не сходится [']. Нас интересует вопрос: всегда ли можно выбрать координатную систему так, чтобы невязка сходилась при любых $f \in H$ ? Известно $\left[{ }^{3-5}\right]$, что невязка в методе Ритца сходится, если существует сходный с $A$ самосопряженный положительно определенный оператор $B$, причем $B^{-1}$ вполне непрерывен, а за координатную систему взяты собственные элементы опера- 
тора $B$. Сходный с $A$ оператор $B$ с вполне непрерывным обратным $B^{-1}$ существует тогда и только тогда, когда вложение $D(A) \subset H$ вполне непрерывно, поэтому доказываемая ниже теорема 1 касается прежде всего некомпактного вложения $D(A) \subset H$.

Частным случаем теоремы Н. И. Польского $\left[{ }^{6,7}\right]$ является

Л ем м а 1. Невязка в методе Ритца сходится при любой правой части тогда и только тогда, когда для некоторого $c>0$ выполняется оценка

$$
\left\|P_{n} A x\right\| \geqslant c\|A x\| \quad \forall x \in H_{n}, \quad n=1,2, \ldots .
$$

Т е о р е м а 1. Для любого самосопряженного положительно определенного оператора существует координатная система такая, ито невязка в методе Ритца сходится при любой правой части.

Доказательство. Оператор $A$ по предположению положительно определен, пусть $(A x, x) \geqslant \alpha\|x\|^{2}(\alpha>0) \quad \forall x \in D(A)$. Пусть $E_{\lambda}-$ спектральное разложение оператора $A$ (см., напр., $\left.\left[{ }^{8}\right]\right)$. Введем разбиение полуоси $[\alpha, \infty): \alpha=\alpha_{0}<\alpha_{1}<\ldots<\alpha_{i}<\ldots, \alpha_{i} \rightarrow \infty$. Тогда $H$ распадается на ортогональную сумму подпространств $H^{\alpha}=$ $=\left(E_{\alpha i}-E_{\alpha_{t-1}}\right) H, i=1,2, \ldots$. Подпространства $H^{\alpha}$ инвариантны относительно $A$ и сужения $A$ на эти подпространства ограничены $\left(\alpha_{i-1}\|x\|^{2} \leqslant(A x, x) \leqslant \alpha_{i}\|x\|^{2}, x \in H^{\alpha}\right)$. Выберем в каждом из подпространств $H^{\alpha} i$ полную ортонормированную систему $\varphi_{i j}, j=1,2, \ldots$ (поскольку $H^{\alpha \imath}$ могут быть бесконечномерными, эти базисы в общем бесконечны). Объединим элементы $\varphi_{i j}$ в общую последовательность $\left\{\varphi_{k}\right\}_{1}{ }^{\infty}$, например, в порядке возрастания суммы индексов: $\varphi_{1}=\varphi_{11}, \varphi_{2}=\varphi_{12}$, $\varphi_{3}=\varphi_{21}, \ldots$.

Покажем сначала, что координатная система $\left\{\varphi_{k}\right\}$ полна в $D(A)$. Пусть элемент $x \in D(A)$ такой, что $\left(x, \varphi_{i j}\right)_{D(A)}=0 \forall i, j$. Имеет место

$$
\|A x\|^{2}=\sum_{i=1}^{\infty}\left\|A\left(E_{\alpha_{1}}-E_{\alpha_{l-1}}\right) x\right\|^{2} .
$$

Далее,

$$
\left\|A\left(E_{\alpha_{l}}-E_{\alpha_{l-1}}\right) x\right\|^{2}=\left(A x, A\left(E_{\alpha_{1}}-E_{\alpha_{l-1}}\right) x\right) .
$$

Пусть $\left(E_{\alpha_{\ell}}-E_{\alpha_{\ell-1}}\right) x=\sum_{j=1}^{\infty} \xi_{j} \varphi_{i j}, \quad$ ряд сходится в $H^{\alpha_{t}}$. Тогда, поскольку на $H^{\alpha}$ оператор $A$ ограничен,

$$
\left(A x, A\left(E_{\alpha_{l}}-E_{\alpha_{l-1}}\right) x\right)=\left(A x, \sum_{j=1}^{\infty} \xi_{j} A \varphi_{i j}\right)=\sum_{j=1}^{\infty} \xi_{j}\left(A x, A \varphi_{i j}\right)=0 .
$$

Этим показано, что $A x=0$, значит, $x=0$ и координатная система $\left\{\varphi_{h}\right\}$ полна в $D(A)$.

Возьмем теперь числа $\alpha_{i}$ через равные промежутки длиной $\varepsilon$, т. е. $\alpha_{i}=\alpha+i \varepsilon, i=1,2, \ldots$. Пусть $x \in H_{n}$; напомним, что $H_{n}-$ линейная оболочка элементов $\varphi_{1}, \ldots, \varphi_{n}$. Рассмотрим разложение $x=\sum_{i=1}^{m} x_{i}$, где $x_{i} \in H^{\alpha}$. Отметим, что $x_{i} \in H_{n}$. Тогда $\left(P_{n} A x, A x\right)=\sum_{i=1}^{m}\left(P_{n} A x_{i}, A x_{i}\right)$, поскольку $A x_{i}, P_{n} A x_{i} \in H^{\alpha}$, а подпространства $H^{\alpha} t$ попарно ортогональны. Выберем числа $\mu_{i} \in[\alpha+(i-1) \varepsilon, \alpha+i \varepsilon], i=1, \ldots, m$. Имеем

$$
\left(P_{n} A x_{i}, A x_{i}\right)=\left(P_{n}\left(\left(A-\mu_{i} I\right) x_{i}+\mu_{i} x_{i}\right), \quad\left(A-\mu_{i} I\right) x_{i}+\mu_{i} x_{i}\right)=
$$




$$
\begin{gathered}
=\mu_{i}^{2}\left\|x_{i}\right\|^{2}+\left(P_{n}\left(A-\mu_{i} I\right) x_{i}, \mu_{i} x_{i}\right)+\left(\mu_{i} x_{i},\left(A-\mu_{i} I\right) x_{i}\right)+ \\
+\left(P_{n}\left(A-\mu_{i} I\right) x_{i},\left(A-\mu_{i} I\right) x_{i}\right)
\end{gathered}
$$

при этом использовано то обстоятельство, что $P_{n} x_{i}=x_{i}$. Поскольку $\left\|A-\mu_{i} I\right\| \leqslant \varepsilon$ в подпространстве $H^{\alpha \iota}$, то отсюда получаем оценку

$$
\left(P_{n} A x_{i}, A x_{i}\right) \geqslant \mu_{i}^{2}\left\|x_{i}\right\|^{2}-2 \varepsilon \mu_{i}\left\|x_{i}\right\|^{2}-\varepsilon^{2}\left\|x_{i}\right\|^{2} .
$$

Аналогично, разлагая

$$
\left(A x_{i}, A x_{i}\right)=\left(\left(A-\mu_{i} I\right) x_{i}+\mu_{i} x_{i},\left(A-\mu_{i} I\right) x_{i}+\mu_{i} x_{i}\right),
$$

получим

$$
\mu_{i}^{2}\left\|x_{i}\right\|^{2} \geqslant\left(A x_{i}, A x_{i}\right)-2 \varepsilon \mu_{i}\left\|x_{i}\right\|^{2}-\varepsilon^{2}\left\|x_{i}\right\|^{2}
$$

следовательно,

$$
\left(P_{n} A x_{i}, A x_{i}\right) \geqslant\left(A x_{i}, A x_{i}\right)-4 \varepsilon \mu_{i}\left\|x_{i}\right\|^{2}-2 \varepsilon^{2}\left\|x_{i}\right\|^{2} .
$$

Далее, из равенства

$$
\left(A x_{i}, x_{i}\right)=\left(\left(A-\mu_{i} I\right) x_{i}+\mu_{i} x_{i}, x_{i}\right)=\mu_{i}\left\|x_{i}\right\|^{2}+\left(\left(A-\mu_{i} I\right) x_{i}, x_{i}\right)
$$

вытекает оценка

$$
\left(A x_{i}, x_{i}\right) \leqslant \mu_{i}\left\|x_{i}\right\|^{2}+\varepsilon\left\|x_{i}\right\|^{2}
$$

значит,

$$
\left(P_{n} A x_{i}, A x_{i}\right) \geqslant\left(A x_{i}, A x_{i}\right)-4 \varepsilon\left(A x_{i}, x_{i}\right)-6 \varepsilon^{2}\left\|x_{i}\right\|^{2}, \quad i=1, \ldots, m .
$$

Учитывая, что

$$
\sum_{i=1}^{m}\left(A x_{i}, A x_{i}\right)=\|A x\|^{2}, \quad \sum_{i=1}^{m}\left(A x_{i}, x_{i}\right)=(A x, x), \quad \sum_{i=1}^{m}\left\|x_{i}\right\|^{2}=\|x\|^{2},
$$

имеем

$$
\left(P_{n} A x, A x\right) \geqslant\|A x\|^{2}-4 \varepsilon(A x, x)-6 \varepsilon^{2}\|x\|^{2} .
$$

Наконец, поскольку $(A x, x) \leqslant\|A x\|\|x\|$ и $\|x\| \leqslant \alpha^{-1}\|A x\|$, то

$$
\left(P_{n} A x, A x\right) \geqslant\left(1-\frac{4 \varepsilon}{\alpha}-\frac{6 \varepsilon^{2}}{\alpha^{2}}\right)\|A x\|^{2} .
$$

При достаточно малом числе $\varepsilon$ отсюда вытекает условие (2) и, по лемме 1 , невязка в методе Ритца сходится, если за координатную систему взять $\left\{\varphi_{k}\right\}$.

Теорема доказана.

2. Пусть $V$ и $H$ - сепарабельные гильбертовы пространства, причем $V$ непрерывно вложено в $H$ и плотно в нем. Отождествляя $H$ с его антидвойственным пространством и обозначая через $V^{\prime}$ пространство, антидвойственное к $V$, получаем $V \subset H \subset V^{\prime}$, где каждое пространство плотно в последующем. Если $f \in V^{\prime}$ и $v \in V$, то $(f, v)$ обозначает значение функционала $f$ на $v$ и совпадает со скалярным произведением в $H$ для $f \in H$. Пусть в $V$ задана полная линейно независимая система $\left\{\varphi_{k}\right\}_{1}^{\infty}$, следовательно, она полна также в пространствах $H$ и $V^{\prime}$. Линейные оболочки систем $\left\{\varphi_{k}\right\}_{1}{ }^{n}$, снабженные нормами из $V$ и $H$, обозна- 
чим через $V_{n}$ и $H_{n}$, а если будем рассматривать их как пространства, антидвойственные к $V_{n}$, - через $V_{n}^{\prime}$. Итак,

$$
\|v\|_{V_{n^{\prime}}}=\sup _{x \in V_{n},\|x\|_{v}=1}(v, x) \leqslant \sup _{x \in V,\|x\|_{V}=1}(v, x)=\|v\|_{V^{\prime}} \quad \forall v \in V_{n} .
$$

В силу конечномерности рассматриваемых линейных оболочек существуют постоянные $c_{n}$ (пусть они будут минимальными) такие, что

$$
\|v\|_{V^{\prime}} \leqslant c_{n}\|v\|_{V_{n^{\prime}}} \quad \forall v \in V_{n}, \quad n=1,2, \ldots .
$$

Пусть $P_{n}$ - ортопроектор в $H$, проектирующий на $H_{n}$. Оператор $P_{n}$ ограничен единицей и как оператор из $V^{\prime}$ в $V_{n}^{\prime}$. Его расширение по непрерывности на $V^{\prime}$ также будем обозначать через $P_{n}$, при этом $\left\|P_{n}\right\|_{V^{\prime} \rightarrow V_{n}} \leqslant 1$.

Л ем м а 2. Справедливы равенства

$$
c_{n}=\left\|P_{n}\right\|_{V \rightarrow V}=\left\|P_{n}\right\|_{V^{\prime} \rightarrow V^{\prime}}, \quad n=1,2, \ldots .
$$

Д ок аз ат ель с т о. Выберем $v \in V_{n}$. Тогда имеем

$$
\begin{aligned}
\|v\|_{V^{\prime}} & =\sup _{x \in V,\|x\|_{V}=1}(v, x)=\sup _{x \in V,\|x\|_{V}=1}\left(v, P_{n} x\right) \leqslant \\
& \leqslant\|v\|_{V_{n^{\prime}}} \sup _{x \in V,\|x\|_{V}=1}\left\|P_{n} x\right\|_{V}=\left\|P_{n}\right\|_{V \rightarrow V}\|v\|_{V_{n^{\prime}}},
\end{aligned}
$$

следовательно, $\quad c_{n} \leqslant\left\|P_{n}\right\|_{V \rightarrow V}$.

Так как

$$
\begin{aligned}
\left\|P_{n} v\right\|_{V} & =\sup _{x \in V^{\prime},\|x\|_{V^{\prime}=1}}\left(x, P_{n} v\right)=\sup _{x \in V^{\prime},\|x\|_{V^{\prime}=1}}\left(P_{n} x, v\right) \leqslant \\
& \leqslant \sup _{x \in V^{\prime},\|x\|_{V^{\prime}=1}}\left\|P_{n} x\right\|_{V^{\prime}}\|v\|_{V} \quad \forall v \in V,
\end{aligned}
$$

то $\left\|P_{n}\right\|_{V \rightarrow V} \leqslant\left\|P_{n}\right\|_{V^{\prime} \rightarrow V^{\prime}}$.

Наконец, из

$$
\left\|P_{n} v\right\|_{V^{\prime}} \leqslant c_{n}\left\|P_{n} v\right\|_{V_{n^{\prime}}} \leqslant c_{n}\left\|P_{n}\right\|_{V^{\prime} \rightarrow V_{n^{\prime}}}\|v\|_{V^{\prime}} \leqslant c_{n}\|v\|_{V^{\prime}} \quad \forall v \in V^{\prime}
$$

следует, что $\left\|P_{n}\right\|_{V^{\prime} \rightarrow V^{\prime}} \leqslant c_{n}$.

Лемма доказана.

Равенством $(u, v)_{v}=(\Lambda u, v)=(\Lambda u, \Lambda v)_{v^{\prime}} \forall u, v \in V$ определяется оператор $\Lambda$, который является изоморфизмом из $V$ на $V^{\prime}$, а в пространстве $V^{\prime}$ - самосопряженным и положительно определенным с областью определения $D(\Lambda)=V$ оператором. Его энергетическое пространство есть $H$. Решим уравнение

$$
\Lambda u=f,
$$

где $f \in V^{\prime}$, методом Ритца, используя координатную систему $\left\{\varphi_{k}\right\}$.

Т еорем а 2. Последовательность $c_{n}$ в неравенствах (3) ограничена тогда и только тогда, когда невязка в методе Ритиа для решения уравнения (4) сходится при всех $f \in V^{\prime}$.

Действительно, приближением по Ритцу является $u_{n}=P_{n} u$ и сходимость невязки равносильна сходимости $u_{n} \rightarrow u$ в $D(\Lambda)=V$ или $P_{n} u \rightarrow u$ в $V$ при всех $u \in V$. А последнее, по теореме Банаха-Штейнгауза, равносильно равномерной ограниченности операторов $P_{n}$ в $V$ или, по лемме 2 , ограниченности последовательности $c_{n}$.

Из теорем 1 и 2 следует, что в пространстве $V$ существует полная 
координатная система такая, что последовательность $c_{n}$ в (3) ограничена.

Ограниченность последовательности $c_{n}$ важна при решении эволюционных уравнений методом Галеркина. Именно тогда удается доказать сходимость приближенных решений в норме пространства решений рассматриваемого эволюционного уравнения с двусторонними оценками $\left[{ }^{9,10}\right]$. Использование координатной системы с ограниченной последовательностью $c_{n}$ позволяет доказать существование решения уравнений параболического типа с операторами локального типа (эта проблема будет рассмотрена отдельно). Такая координатная система также может быть предложена вместо собственных элементов оператора $\Lambda$ при доказательстве существования решения некоторых нелинейных краевых задач $\left[{ }^{11}\right]$, чтобы избавиться от компактности вложения $V \subset H$.

\section{Л И Т Е Р А Т У Р А}

1. В а й н икко Г. М., У м а н ски й Ю. Б., Функц. анализ и его прилож., 2, № 2, $87-88$ (1968).

2. Михлин С. Г., Проблема минимума квадратичного функционала, М.-Л., ГИТТЛ, 1952.

3. Михлин С. Г., Докл. АН СССР, 106, № 3, 391-394 (1956).

4. В а й н и к о Г., М., Докл. АН СССР, 179, № 5, 1029-1031 (1968).

5. Михлин С. Г., Вариационные методы в математической физике, М., «Наука», 1970.

6. По льс к и й Н. И., Докл. АН СССР, 143, № 4, 787-790 (1962).

7. К р а сносельский М. А., В айниккоГ. М., 3 аб рейко П. П., Рутиц ки й Я. Б., Стеценко В. Я., Приближенное решение операторных уравнений, М., «Наука», 1969.

8. Р и с с Ф., С ёк е фа ль в и - Н а д ь Б., Лекции по функциональному анализу, М., «Мир», 1979.

9. В а й н и к к о Г. М., О я П. Э., Дифференц. уравнения, 11, № 7, 1269-1277 (1975).

10. О я П., Уч. зап. Тартуск. ун-та, $342,237-248$ (1974).

11. Ли он с Ж.-Л., Некоторые методы решения нелинейных краевых задач, М., «Мир», 1972.

Тартуский государственный университет

Поступила в редакцию $27 /$ III 1981

\section{KOORDINAATSUSTEEMI VALIKUST RITZI MEETODILE JA EVOLUTSIOONIVORRANDITE LAHENDAMISEL GALJORKINI MEETODILE}

On näidatud, et igale tōkestamata, positiivselt määratud enesekaasoperaatorile $A$ leidub selline koordinaatsüsteem, mille puhul vōrrandi $A u=f$ lahendamiseks kasutatava Ritzi meetodi jääkliige koondub iga vabaliikme $f$ korral. Seda koordinaatsüsteemi saab kasutada lokaalset tüüpi operaatoritega evolutsioonivõrrandite ja mōningate mittelineaarsete võrrandite lahendi olemasolu tõestamiseks. Samuti saab sellise koordinaatsüsteemi puhul anda Galjorkini meetodil lahendatavate lineaarsete evolutsioonivõrrandite koonduvuskiirust iseloomustavad kahepoolsed veahinnangud. 


\section{P. OIA}

\section{SUR LE CHOIX DU SYSTEMME DE COORDONNEES A LA METHODE DE RITZ ET A LA MÉTHODE DE GALERKINE POUR LES ÉQUATIONS D'ÉVOLUTION}

La convergence de la méthode de Ritz pour l'équation stationnaire $A u=f$ est très étudiée. On sait que la suite des solutions approchées $u_{n}$ trouvées par la méthode de Ritz converge vers la solution $u$ dans l'espace de Friedrichs $H_{A}$ quel que soit $f$ lorsque le système de coordonnées est complet dans $H_{A}$. On sait aussi [ ${ }^{1}$ ] que pour tout opérateur A non borné autoadjoint positivement défini il est toujours possible de choisir un système de coordonnées complet dans $D(A)$ et un élément $f$ tels que le reste $A u_{n}-f$ ne tend pas vers zéro. Cependant, nous montrons que, dans les mêmes hypothèses sur $A$, il existe un système de coordonnées où la méthode de Ritz donne le reste tendant vers zéro pour tout $f$. A cet effet, nous avons recours à la décomposition spectrale de l'opérateur $A$.

Considérons deux espaces de Hilbert séparables $V$ et $H$ tels que: $V$ est inclus dans $H, V$ est dense dans $H$. Nous identifions $H$ et son antidual $H^{\prime}$. Si $V^{\prime}$ désigne l'antidual de $V$, nous avons les inclusions $V \subset H \subset V^{\prime}$, les injections étant continues, chaque espace étant dense dans le suivant. Nous noterons par $(\cdot, \cdot)$ le produit scalaire dans $H$ ou dans l'antidualité entre $V$ et $V^{\prime}$. Soit $\left\{\varphi_{k}\right\}$ un système de coordonnées linéairement indépendant complet dans $V$. Le sous-espace engendré par des éléments $\varphi_{1}, \ldots, \varphi_{n}$ muni de la norme de $V$ sera noté par $V_{n}$. Nous considérons aussi l'antidual de $V_{n}$ désigné par $V_{n}^{\prime}$. Donc,

$$
\|v\| V_{n^{\prime}}=\sup _{x \in V_{n},\|x\|_{v}=1}(v, x) \leqslant \sup _{x \in V,\|x\|_{v}=1}(v, x)=\|x\|_{v^{\prime}} \forall v \in V_{n} .
$$

Ces espaces étant de dimension finie il existe des constantes $\mathrm{c}_{n}$ (choisissons-en les plus petites possibles) telles que

$$
\|v\|_{v^{\prime}} \leqslant c_{n}\|v\|_{V_{n}} \quad \forall v \in V_{n}, \quad n=1,2, \ldots .
$$

L'égalité $(u, v)_{v}=(\Lambda u, v)=(\Lambda u, \Lambda v)_{V^{\prime}} \forall u, v \in V$ définit un opérateur $\Lambda$ autoadjoint positivement défini dans $V^{\prime}$. Appliquons maintenant la méthode de Ritz par le système $\left\{\varphi_{k}\right\}$ à l'équation $\Lambda u=f, f \in V^{\prime}$. Nous voyons qu'en ce cas le reste tend vers zéro si et seulement si la suite $c_{n}$ est bornée. Par conséquent, il existe un système de coordonnées complet dans $V$ avec la suite $c_{n}$ bornée. Ce système permet de démontrer l'existence de la solution des équations d'évolution avec les opérateurs de type local et d'établir pour la méthode de Galerkine des estimations de convergence en deux sens. Et tous cela sans supposition de la compacité de l'injection $V \subset H$. 